\title{
Memory Devices via Unipolar Resistive Switching in Symmetric Organic-Inorganic Perovskite Nanoscale Heterolayers
}

\author{
Xianxi Yu, ${ }^{\perp}$ Tangyao Shen, ${ }^{\perp}$ Chunqin Zhu, ${ }^{\perp}$ Qi Zeng, Anran Yu, Shaobo Liu, Ruichen Yi,
} Zhenhua Weng, Yiqiang Zhan, Xiaoyuan Hou, and Jiajun Qin*

Cite This: ACS Appl. Nano Mater. 2020, 3, 11889-11896

Read Online

\section{ACCESS | Lill Metrics \& More | 回 Article Recommendations ｜（） Supporting Information}

ABSTRACT: Organic-inorganic hybrid perovskite thin films with nanostructured polycrystalline grains have shown great potential in various nanoscale optoelectrical applications. Among them, the field of electrical memory has fallen behind due to insufficient knowledge of the related resistive switching characters and mechanisms. In the present work, switching behaviors of perovskite memory devices are systematically analyzed by comparing them with organic memory devices. We found that decreasing the conductivity of a polycrystalline perovskite thin layer would lead to unipolar switching behaviors, which is supplementary to the present perovskite memory family where bipolar switching is

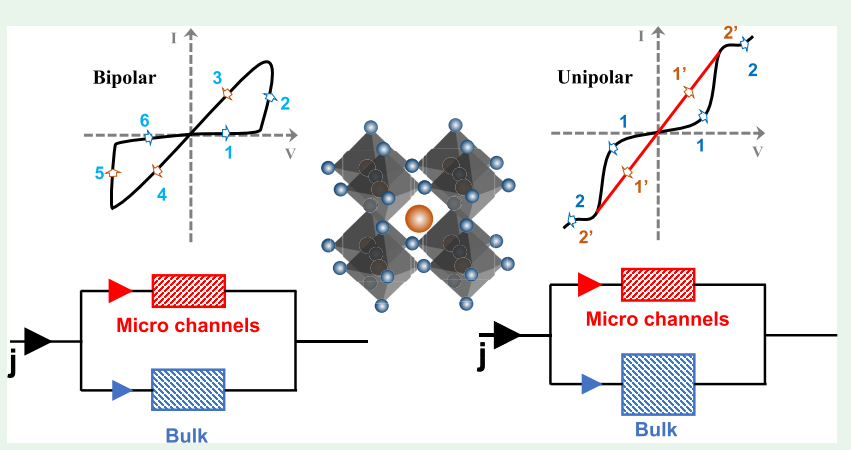
commonly reported. Moreover, our proposed symmetrical device with a nanoscale heterolayer structure enables us not only to achieve highly reproducible unipolar switching devices but also to settle the argument whether microconducting channels exist within perovskite memory devices through characterizing the microscopic morphological homogeneity. Surprisingly, the scanning electron microscopy results show that partial $10 \mu \mathrm{m}$ large perovskite grains would be decomposed into various $100 \mathrm{~nm}$ small grains under high external bias, indicating the presence of microconducting channels. Furthermore, energy-dispersive X-ray spectroscopy results together with photoluminescence results of the perovskite thin film before and after applying bias are nearly identical, demonstrating that microconducting channels are formed without any difference in compositions or optical properties. Our discoveries provide a practical strategy to achieve electrical storage via organicinorganic hybrid perovskite thin-film devices.

KEYWORDS: perovskite memory, resistive switching, unipolar switch, bipolar switch, microconducting channels

\section{INTRODUCTION}

The superior optoelectrical properties enable organicinorganic hybrid perovskites to be almighty materials with applications in various fields such as photovoltaics, ${ }^{1,2}$ lightemitting diodes, ${ }^{3-5}$ photodetectors, ${ }^{6,7}$ and lasers. ${ }^{8,9}$ However, their application in the nanoscale electrical memory devices has fallen behind due to insufficient knowledge of perovskite switching as compared with organic memory devices. Considering the electrical switching behavior where the device can be switched between a low-resistance state ( $\mathrm{ON}$ state) and a high-resistance state (OFF state) during the current-voltage $(I-V)$ scan, the switch $\mathrm{ON}$ process refers to the transition from an OFF state to an ON state, while the switch OFF process refers to the transition from the ON state to the OFF state. $^{10,11}$ Especially, according to the switch $\mathrm{ON}$ and switch OFF history during $I-V$ scan, the memory devices are usually clarified into three types: unipolar switching type, ${ }^{12}$ bipolar switching type, ${ }^{13}$ and write-once-read-many times (WORM) switching type. ${ }^{14}$ It should be noted that in organic-inorganic hybrid perovskite devices, the WORM switching type is usually observed when device resistance goes down instantly under certain bias and never goes up in the following scans. ${ }^{14,15}$ In this kind of switch, the OFF state can be switched to the ON state and the device remains in the $\mathrm{ON}$ state forever. Thus, the device can only be switched $\mathrm{ON}$ once and can never be switched OFF. Especially, many perovskite memory devices show bipolar switching type, ${ }^{16-23}$ where the devices can be switched ON under a forward bias and can only be switched OFF by a reverse bias. However, the unipolar switching type with switch $\mathrm{ON}$ and switch OFF processes occurring in the same bias direction has seldom been reported in the perovskite memory. ${ }^{24}$ It is quite different from organic memory devices with commonly observed unipolar switching type when the conductance of the active layer was decreased. ${ }^{10,11}$ As the promising candidate for future perovskite memory applica-

Received: September 9, 2020

Accepted: November 25, 2020

Published: December 7, 2020 


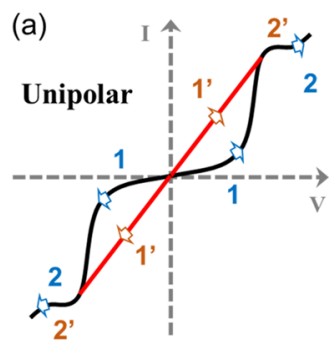

Start from OFF state: $1 \rightarrow 2$ Start from ON state: $1^{\prime} \rightarrow 2$

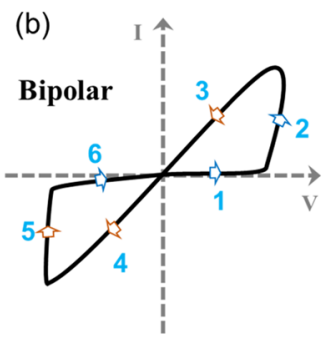

Scan direction: $1 \rightarrow 6$

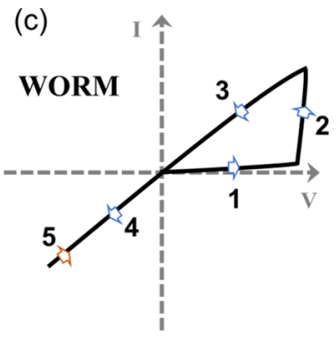

Scan direction: $1 \rightarrow 5$
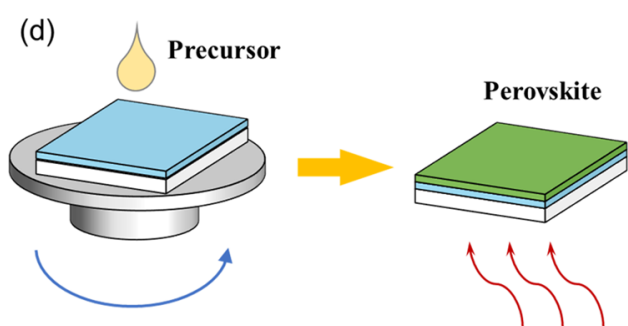

3D

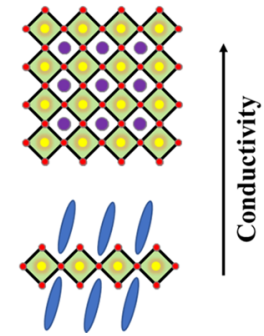

Figure 1. Schematic diagrams to show the $I-V$ curves of three different resistive switching types and the perovskite film processing. (a) Unipolar switching with switch ON and switch OFF occurring in the same bias direction. The device is in OFF and ON states before scan process 1 and process $1^{\prime}$, respectively. (b) Bipolar switching with switch ON occurring at forward bias (process 2) and switch OFF occurring at reverse bias (process 5). (c) WORM switching with switch ON at forward bias (process 2) but no switch OFF process. (d) Schematic diagram showing perovskite thin-film fabrication through spin coating. Note that, $2 \mathrm{D}$ and 3D perovskite thin films with different conductivities can be obtained through changing the compositions in the precursor solution.

tions, it is highly desirable to develop unipolar switching, which enables an easier read-write process through the samedirectional operation. Moreover, the $I-V$ characteristics under both forward and reverse scans are usually symmetrical, which brings about more flexibility in memory programming. The lack of a unipolar switching in the perovskite memory family indicates some unrevealed characters of hybrid perovskites as compared with organics, which deserves further understanding.

Besides, the switching mechanism in perovskite memory is under debate. Wang et al. proposed that trapping/detrapping is responsible for the switch $\mathrm{ON}$ and switch OFF processes in an $\mathrm{Au} / \mathrm{CH}_{3} \mathrm{NH}_{3} \mathrm{PbI}_{3-x} \mathrm{Cl}_{x} / \mathrm{FTO}$ device, ${ }^{25}$ which refers that the carrier conduction in both $\mathrm{ON}$ and OFF states is through the device homogeneously. However, some other groups attributed the switch ON process to the formation of microconducting channels. For example, Jung et al. claimed that microchannels are formed through metal atoms in a $\mathrm{Pt} / \mathrm{Ti}$ / perovskites/PMMA/Ag device, ${ }^{26}$ which is similar to organic memory. In addition, Lee et al. claimed that the channels were formed by a halide vacancy in an $\mathrm{Au} / \mathrm{CH}_{3} \mathrm{NH}_{3} \mathrm{PbI}_{3} / \mathrm{ITO}$ device, ${ }^{16}$ while Ren et al. supposed the channels to be from halide ions in an $\mathrm{Au} / 2 \mathrm{D}$ perovskite/graphene device. ${ }^{18}$ To settle down the arguments on the mechanism responsible for perovskite memory, it is highly suggested to provide direct evidence of microconducting channels and their composition.

In the present work, we proposed a symmetrical device structure with two identical nanoscale heterolayer units (ITO/ perovskite) attached to each other to study the mechanism of perovskite memory. This device structure enables us to directly characterize the nanoscale morphological change of the perovskite layer after an electrical operation. Interestingly, unipolar switching type was observed together with partially perovskite grain decomposition, which directly verifies the existence of microconducting channels within perovskite memory devices. Moreover, the nearly unchanged composition and photoluminescence (PL) spectra further indicate that the microchannels may not originate from halide ions. The proposed microconducting channels model is beneficial for the application of perovskite memory.

\section{RESULTS AND DISCUSSION}

Different Switching Types of Memory Device. As for memory devices including hybrid perovskite memory, the switching behaviors can be clarified into three types, with the temporal $I-V$ curves are schematically shown in Figure 1. Specifically, the unipolar switching type shows nearly identical switching behaviors under operations of positive bias only and negative bias only. As we can see from Figure 1a, a scan from the initial OFF state leads to very low current at low bias (process 1), and it is switched $\mathrm{ON}$ with the current increasing significantly at a certain higher bias (the threshold voltage). After switching ON, the device shows high current at low bias, indicating the $\mathrm{ON}$ state (process $1^{\prime}$ ). If the bias is increased to a much higher value than the threshold voltage (processes 2 and $2^{\prime}$ ), the device will be switched OFF. It should be noted that switch ON and switch OFF processes can occur at the same-directional bias, which is the key character of unipolar switching-type memory devices. On the other hand, the bipolar switching type is depicted in Figure $1 \mathrm{~b}$ by following the scanning loop from a positive bias to a negative bias (shown as processes from 1 to 6 ). The device shows the OFF state with low current at low bias (process 1) initially and is switched $O N$ under forward bias (process 2). Once the device is switched $\mathrm{ON}$, it cannot be switched OFF through any forward-bias operations. The switching OFF process only occurs when a relatively high reverse bias is applied (shown as process 5). Additionally, the WORM switching-type device shows switch ON once and cannot be switched OFF in Figure 1c. Note that, the spin-coating method enables easy tuning of perovskite films with different conductivities (Figure 1d), which provides the 
(a)

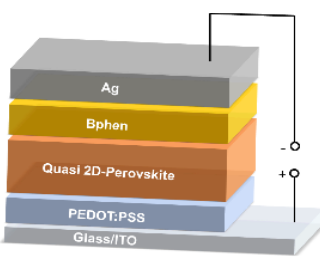

(b)

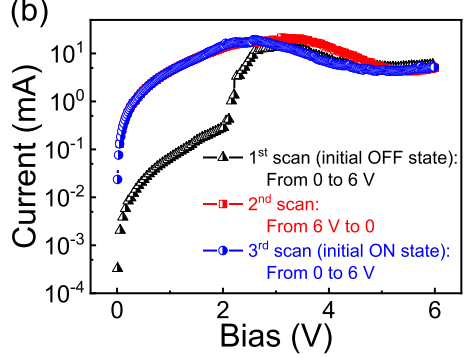

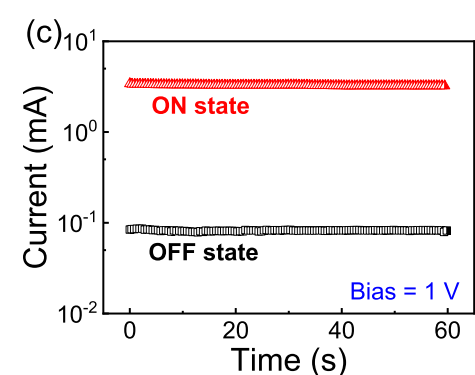

Figure 2. Characterizations of a unipolar switching-type perovskite memory device. (a) Schematic diagram of the device structure: ITO/ PEDOT:PSS/quasi-2D perovskite film/Bphen $(20 \mathrm{~nm}) / \mathrm{Ag}$. (b) $I-V$ curves under three different scans: from 0 to $6 \mathrm{~V}$ with an initial OFF state (black triangles) and from 6 to $0 \mathrm{~V}$ (red squares) and from 0 to $6 \mathrm{~V}$ with an initial ON state (blue circles). (c) Stability test of ON (red curve) and OFF (black curve) states at $1 \mathrm{~V}$.

(a)
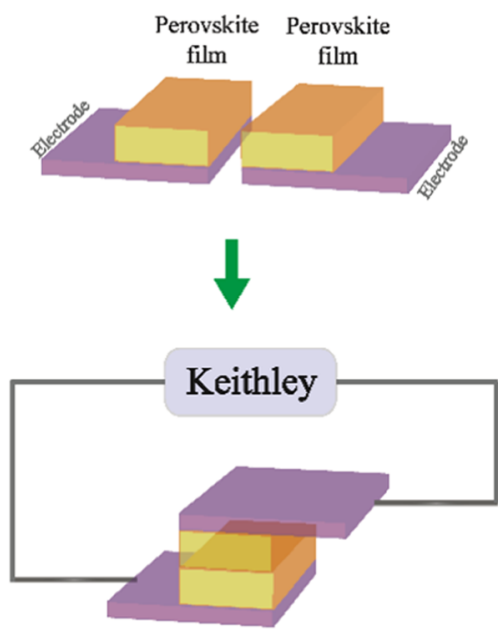

(b)
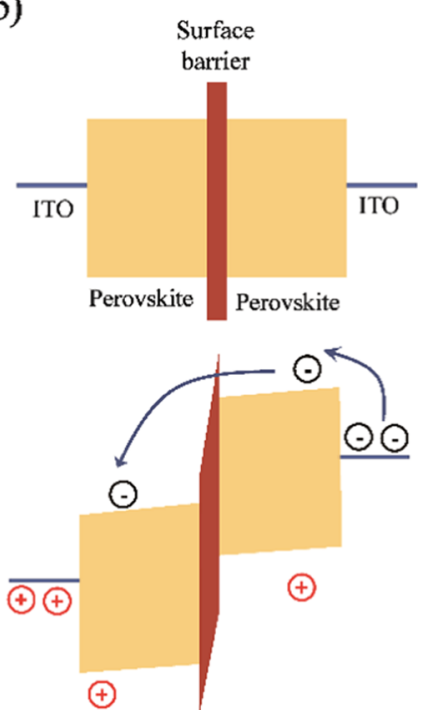

Figure 3. Cartoons and energy diagrams of our symmetrical device. (a) Cartoons of the device preparation procedures. (b) Energy diagrams of the device without (upper figure) and with (bottom figure) biases.

opportunity to achieve the mentioned three types of memory devices.

Unipolar Switching by Incorporating Insulating Ligands. It is worth pointing out that bipolar switching type has been widely reported, while the unipolar one is seldom discussed in perovskite memory devices. According to our previous understanding of organic memory devices, ${ }^{27}$ bipolar switching type can be discovered in devices with relatively good conductivity, while a unipolar switch can be observed in devices with relatively poor conductivity (see detailed examples in Table S1 for conductivity comparison). Especially, excellent carrier transport is easily achieved in hybrid perovskites, which might be the origin of commonly seen bipolar switching type memory devices. ${ }^{16-19}$ Here, the question arises whether the unipolar switching phenomenon will occur in perovskite devices when the conductivity is decreased. Herein, to decrease the conductivity of the perovskite layer in the device, we added insulating long-chain ligands $\mathrm{PEABr}$ into the perovskite film, resulting in the quasi2D perovskite films with carrier transport blocking ligands distributed everywhere. ${ }^{28}$ Amazingly, quite stable unipolar switching type is realized in the device with the architecture of ITO/PEDOT:PSS/perovskite/Bphen $(20 \mathrm{~nm}) / \mathrm{Ag}$ (in Figure $2 \mathrm{a})$. As shown in Figure $2 \mathrm{~b}$, during the first scan from 0 to $6 \mathrm{~V}$, the device is in the OFF state initially when the bias is below 2
$\mathrm{V}$ (at $1 \mathrm{~V}: I=0.066 \mathrm{~mA}$ ). At around $2.1 \mathrm{~V}$, the current increases sharply, shown as the switch $\mathrm{ON}$ phenomenon. When the bias is further increased to $6 \mathrm{~V}$, the device will be switched OFF, evidenced by the negative differential resistance (NDR) at the region from 3.4 to 5.6 V. If a reverse scan from 6 to $0 \mathrm{~V}$ is applied to the device (the $2 \mathrm{nd}$ scan), the $I-V$ curve of the device also shows NDR in the similar region, indicating that the device is slowly switched ON. After the scan goes below $2 \mathrm{~V}$, the $\mathrm{ON}$ state is realized and cannot be switched OFF at a bias lower than $2 \mathrm{~V}$, leading to a much higher current (at $1 \mathrm{~V}: I=4.24 \mathrm{~mA}$ ) than that in the OFF state. The ON/ OFF ratio is as high as 64 . The following third scan starting from 0 to $6 \mathrm{~V}$ further verifies that the $\mathrm{ON}$ state is stable, where the $I-V$ curve at the region from 0 to $2 \mathrm{~V}$ coincides with that of the second scan. We should point it out that after forward scanning and NDR appears (the first scan), the followed reverse scan from the high bias to the low bias (second scan) will experience turn-on voltage, which will lead to the switch ON process. Therefore, the resulting state in the low bias region will always be the $\mathrm{ON}$ state. If we repeat a forward scan from 0 to high bias after the RESET process with NDR appearing, a high-resistance state can be maintained in the low bias region. Moreover, the stability test is applied at a constant bias of $1 \mathrm{~V}$ as a memory device in Figure 2c. The ON and OFF states are characterized for $60 \mathrm{~s}$, showing current at 3.3 and 
$0.08 \mathrm{~mA}$, respectively. Hence, we prove that the unipolar switching type can be realized by decreasing the conductivity of the perovskite layer, which is similar to organic memory devices.

To confirm that the resistive switching originates from the poor conductivity of our perovskite device, we have fabricated the following control devices: (1) ITO/PEDOT:PSS/Ag, (2) ITO/quasi-2D perovskite film/Ag, and (3) ITO/Bphen/Ag. All of the three devices show similar $I-V$ curves with Ohmic contact property (Figure S1) and no resistive memory behaviors. It verifies that our electrical memory does not originate from transport layers. Specifically for device 2 with a single layer of the perovskite film, the loss of memory may originate from the dominant leak current by the penetration of silver atoms into the perovskite film. Notably, the conductivity of the perovskite film can be increased by decreasing the ratio of long-chain ligands (to the target average $\langle n\rangle$ value to be 10) to obtain the bipolar switching phenomenon. As shown in Figure S2, the device is switched $\mathrm{ON}$ once the bias polarity is reversed, see the process from " 4 " to " 1 " and from " 2 " to " 3 ". The switching OFF process occurs at the end of scan " 1 " and " 3 ". This kind of memory performance is a bipolar switching behavior, which is slightly different from Figure 1b. Similar bipolar $I-V$ characteristics have been reported in our previous work. ${ }^{10}$ We should point out that these bipolar switching behaviors are not so repeatable as compared with unipolar switching behaviors in our experiments.

Symmetrical Device Design and I-V Characterizations. As discussed previously, the switching mechanism remains unsettled on whether microconducting channels exist in the ON state. Thus, direct measurement is required to check the conducting homogeneity of the devices. Here, we propose a new device fabrication strategy that enables us to explore this issue. Figure $3 \mathrm{a}$ shows the schematic diagram of how our devices work. In the fabrication process, we obtain two identical structures with ITO/perovskite unit first. Then, these two units are attached to each other with perovskite surface contacted. After applying a certain pressure to ensure good contacts between two perovskite layer surfaces, $I-V$ characteristics can be obtained by applying external bias on the device with a structure of ITO/perovskite/perovskite/ITO. It is widely reported that the boundary defects originated from dangling bonds and charged defects in polycrystalline perovskite films are detrimental for carrier transport. ${ }^{5,29,30}$ Herein, various efforts have been devoted to passivation of the boundary defects toward better device performance, e.g., additive engineering or the TOPO treatment. ${ }^{30}$ Therefore, the untreated perovskite polycrystalline film surface contains plenty of defects, leading to carrier trapping/blocking sites that will act as an insulating layer at the perovskite/perovskite interface (see Figure $3 b$ ). Therefore, carrier transport can be largely decreased as schematically shown by the energy diagram in the bottom figure of Figure $3 \mathrm{~b}$. In our symmetrical device, the accurate value of our external mechanical pressure in the fabrication cannot be directly detected within the device. However, reproducible device performance can still be achieved through monitoring the current density at low bias. During the characterization, we noticed that too high/low pressure will lead to a short circuit/open circuit, and the normal device $I-V$ (current density-voltage) curve can ensure our reproducible device performance. According to our previous experiment with a less conductive quasi-2D perovskite layer in perovskite memory devices (Figure 2), our proposed strategy is also expected to achieve unipolar switch behaviors. Besides, our symmetrical design enables us to separate the two identical ITO/perovskite units to check the contact morphology before and after applying external bias. It should be noted that this design provides the possibility to directly monitor the conducting homogeneity.

Now, we explore the switching mechanism of perovskite memory based on our symmetrical device design. The 3D perovskite $\left(\mathrm{MAPbBr}_{3}\right)$ film is produced by spin-casting precursor solutions onto ITO substrates. Surprisingly, the device with our symmetrical structure of ITO/MAPbBr 3 / $\mathrm{MAPbBr}_{3} / \mathrm{ITO}$ exhibits excellent unipolar switching behaviors. Figure 4 a presents the $I-V$ curves of different voltage scanning

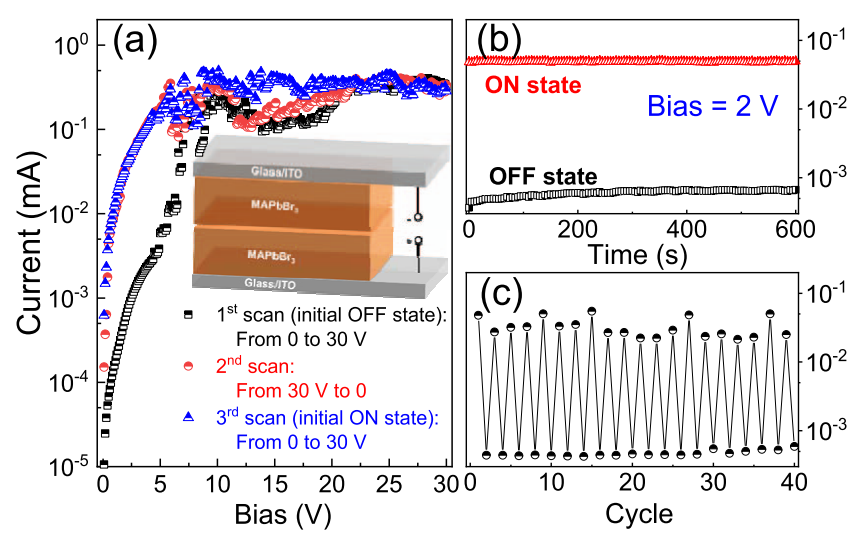

Figure 4. Characterizations of unipolar switching in our perovskite memory device prepared with symmetrical fabrication strategy. (a) I$V$ curves under three different scans: from 0 to $30 \mathrm{~V}$ with an initial OFF state (black squares) and from 30 to $0 \mathrm{~V}$ (red circles) and from 0 to $30 \mathrm{~V}$ with an initial ON state (blue triangles). The inset shows the device structure [ITO/MAPbBr $3 / \mathrm{MAPbBr}_{3} / \mathrm{ITO}$ ]. (b) Stability test of $\mathrm{ON}$ and OFF states at a constant bias of $2 \mathrm{~V}$. (c) Device current at $2 \mathrm{~V}$ at different read-write cycles. In each cycle, the device is switched $\mathrm{ON}$ and OFF alternatively.

pathways (from 0 to $30 \mathrm{~V}$ and from 30 to $0 \mathrm{~V}$ ). Similar to the results of Figure 2a with a device structure of ITO/ PEDOT:PSS/perovskite/Bphen $(20 \mathrm{~nm}) / \mathrm{Ag}$, switching performances are observed with a threshold voltage of $\sim 7.5 \mathrm{~V}$, the $\mathrm{ON}$ state current at $2 \mathrm{~V}$ of $0.036 \mathrm{~mA}$, OFF state current at $2 \mathrm{~V}$ of $6.07 \times 10^{-4} \mathrm{~mA}$, and the ON/OFF ratio of 59.3. I-V characteristics under negative bias also reveal the same unipolar switch behavior with the ON/OFF ratio of around 80 (see Figure S3). The stability test of ON and OFF states at $2 \mathrm{~V}$ is operated for $600 \mathrm{~s}$, as shown in Figure $4 \mathrm{~b}$. It is noted that both currents get only slightly changed, indicating that the $\mathrm{ON} / \mathrm{OFF}$ ratio is always maintained (around 80). Moreover, the read-write experiment is conducted 40 times in Figure $4 \mathrm{c}$ with switching ON and OFF alternatively, which verifies that our device with a symmetrical structure is a highly reproducible unipolar switching-type memory device.

Resistive Switching Mechanism. In the above two unipolar switch-type memory devices (Figures 2 and 4), there is a feature character that NDR will appear after the device is switched $\mathrm{ON}$, i.e., current decreases with increasing bias. According to the theory in organic memory devices, ${ }^{11}$ during this NDR process, the device is competing between the establishing and destroying of conducting microchannels, which is a field-induced process and a current density-driven process, respectively. With bias increasing after threshold 

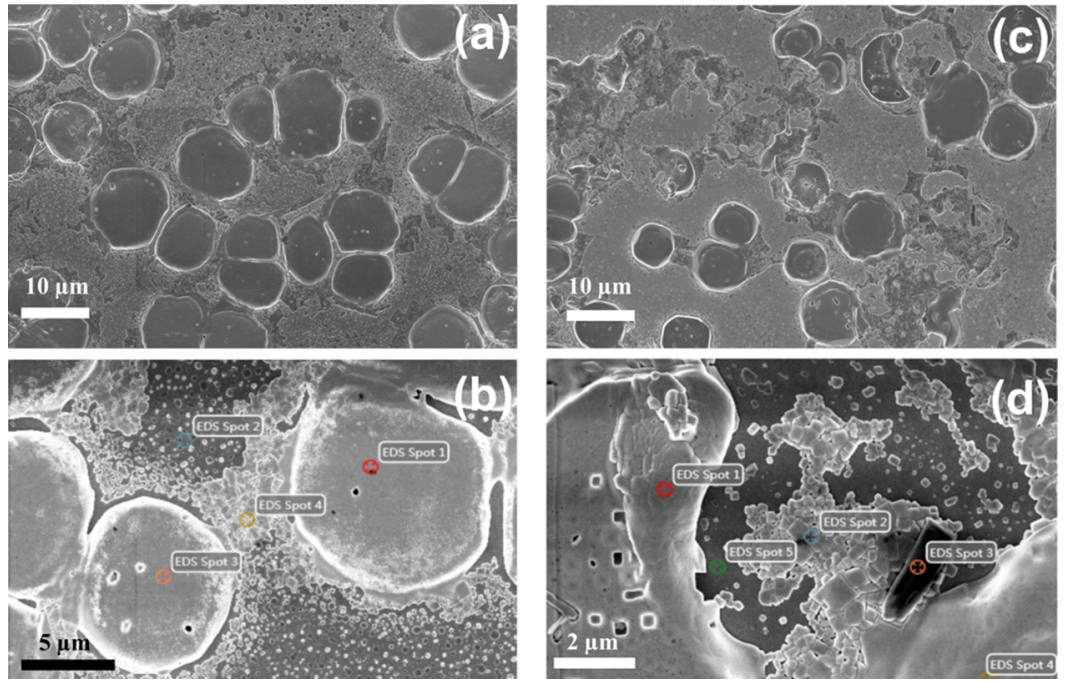

Figure 5. SEM images of perovskite films without and after applying the external bias. (a) Without applying the external bias, the film shows $\sim 10$ $\mu \mathrm{m}$ size large grains locating everywhere. (b) Zoom-in image indicates that the perovskite grains contain smooth edges on top view. (c) After applying the external bias, the anodic perovskite film shows a similar image to (a) but the corners of partial grains are damaged. (d) Zoom-in image to show one damaged corner. The corner is composed of some gathered small grains $(\sim 100 \mathrm{~nm})$.

voltage, establishing the rate of microconducting channels tends to be saturated, while the destroying rate will increase owing to Joule heating. The microconducting channels (with high conductivity) will be totally burnt down at high bias voltage. Thus, the device is switched OFF (reset) from a lowresistance state (LRS) to a high-resistance state (HRS) when the bias exceeds the reset voltage. Specifically, the reset voltages are around $5 \mathrm{~V}$ in Figure $2 \mathrm{~b}$ and $20 \mathrm{~V}$ in Figure $4 \mathrm{a}$ during the $I-V$ scans. To further verify the reset process, the OFF states in Figures $2 c$ and $4 b$ were achieved after applying a bias that exceeds the corresponding reset voltages. On the other hand, the series curves (Figures S4 and S5) extracted from the $I-V$ sweeps in Figures 2 and 4 further show that the resistance follows the same trends with switching $\mathrm{ON}$ and switching OFF processes. Similarly, the resistance goes down quickly after threshold voltages, indicating the switch ON process. On the other hand, at higher bias after the device turns on, the resistance increases, indicating the switch OFF (reset) process. Therefore, the NDR feature in our perovskite memory devices can be well explained by the mechanism of microconducting channels, indicating that the carrier transport is inhomogeneous through the whole perovskite films.

To see whether the carrier transport is homogeneous or not through the whole perovskite film, scanning electron microscopy (SEM) is applied to check the morphological difference between perovskite films before and after applying an external bias. Here, before our SEM characterizations, two identical devices with a device structure of $\left[\mathrm{ITO} / \mathrm{MAPbBr}_{3} /\right.$ $\left.\mathrm{MAPbBr}_{3} / \mathrm{ITO}\right]$ were achieved. Then, the same pressure was applied to these two devices: one without applying an external bias (Figure 5a,b) and the other with an external bias (Figure $5 c, d)$. Obviously, Figure 5a,b shows no morphological changes after experiencing the mechanical pressure, as compared with the pristine-fabricated perovskite film (see Figure S6), exhibiting randomly located large perovskite grains with a size of around $10 \mu \mathrm{m}$. After applying the same-directional external bias, decomposition of partial perovskite grains is observed within the film is coated onto the anodic ITO. Therefore, the morphological difference of these two (Figure $5 a, c)$ originates from the effects of external bias due to the same operations except biasing, indicating that the decomposition of perovskite grains is induced by conducting current passing through. Considering that only partial grains were decomposed at the corners, the current density passing through the whole device would be inhomogeneous, which verifies the existence of microconducting channels. Moreover, energy-dispersive $\mathrm{X}$-ray spectroscopy (EDS) results (Figure S7) do not show any difference between small and large grains, indicating no compositional change during electrical operation. Additionally, the PL results in Figure S8 further verify this claim with nearly no spectrum variance between films before and after applying external bias. To have a better understanding of the morphological change in the film coated onto the anode, SEM results of the cathodic film are also added in Figure S9. Obviously, the cathodic film shows different morphologies on partial grains with some additional layered structures, which indicates that the decomposed grains on the anodic film are recrystallized herein. We should note that the trapping/detrapping process occurs uniformly within the whole film, which cannot explain our observed microconducting channels. Therefore, we proposed that the halide vacancy for the conducting channels is the possible mechanism. Here, owing to the crystalline abruption at the grain boundaries, vacancies are easily formed, serving as microconducting channels. When the current density in the conducting channels is very high, decomposition occurs due to Joule heating, which leads to the switch OFF process.

To unify both a bipolar switch and a unipolar switch through a microconducting channel model, the schematic diagram (Figure S10) is provided to explain the relationship between conductivity and respective switching type. As we can see from the figure, the device current is contributed by both the bulk and the microchannels. With poor conductivity of the bulk, the current passing through the microchannels will be enhanced and may be easier to be burnt down due to Joule heating. Thus, the same-directional bias will lead to the switch OFF process at high voltages, resulting in unipolar switching. Otherwise, when the bulk conductivity is very good at the same-directional bias (for example, positive bias), the shunt current by the bulk will increase, resulting in relatively low 
current density in the microchannels. Thus, these channels will not be burnt down at the same-directional bias. Therefore, bipolar switching is usually observed when the current density through bulk is reduced at reverse bias. This microconducting channels model have been widely observed in organic memory devices. ${ }^{10,27,31}$ As reported, the metal ions are supposed to be the origin of high-conducting channels, through which the current density is much higher than the bulk in the ON state. The burnt down of these channels results in the OFF state. In our perovskite memory devices, since the microconducting channels will lead to high localized current density, serious Joule heating would increase the localized temperature. These heating effects will decompose the perovskite grains on a microscopic scale, resulting in the interruption of microconducting channels. Therefore, the heating effect will be suppressed where recrystallization of perovskite grains occurs.

\section{CONCLUSIONS}

In summary, we compare the perovskite memory devices with organic memory devices and figure out that the lack of unipolar switching in perovskites is attributed to relatively good transport. By incorporating 2D ligands to decrease the conductivity, we can obtain unipolar switching type perovskite memory devices based on the architecture of ITO/ PEDOT:PSS/quasi-2D perovskite/Bphen(20 nm)/Ag. Essentially, using our proposed symmetrical structure with nanoscale heterolayers in $3 \mathrm{D}$ perovskite devices, excellent unipolar switch is also achieved. SEM images provide direct evidence to prove that microconducting channels are responsible for perovskite memory. Therefore, both bipolar switching and unipolar switching in perovskite memory devices can be well explained. Our work provides a supplementary to perovskite memory devices and will be helpful in the understanding of the related working mechanism. Moreover, we provide a practical strategy toward electrical storage via symmetrical nanostructured organic-inorganic hybrid perovskite thin-film devices.

\section{MATERIALS AND METHODS}

Material Processing and Device Fabrication. The ITO substrates were cleaned by ultrasonic treatment with detergent, deionized water, and acetone for $30 \mathrm{~min}$ in each cycle. Then, the ITO substrates were blow dry before UV treatment for $30 \mathrm{~min}$. After that, these substrates would be transferred into a glovebox for symmetrical device fabrication or into a fume hood for PEDOT:PSS preparation.

The precursor solution for quasi-2D perovskite was obtained with $\mathrm{PbAc}_{2} \cdot 3 \mathrm{H}_{2} \mathrm{O} / \mathrm{MABr} / \mathrm{PEABr}=1: 3: 2$ in the DMF solution (with the $\mathrm{Pb}$ source $0.3 \mathrm{M}$ ). The precursor solution for the $\mathrm{MAPbBr}_{3}$ film was obtained with $0.5 \mathrm{M} \mathrm{PbBr} 2,0.5 \mathrm{M} \mathrm{MABr}$, and $10 \mu \mathrm{L}$ of isobutyraldehyde in $1 \mathrm{~mL}$ of DMF. Both solutions were spin-cast in a nitrogen atmosphere at the rate of $3000 \mathrm{rpm}$ for $1 \mathrm{~min}$, followed by annealing at $60{ }^{\circ} \mathrm{C}$ for $30 \mathrm{~min}$.

Bphen and $\mathrm{Ag}$ were thermally deposited under vacuum with thicknesses of 20 and $80 \mathrm{~nm}$. Finally, the perovskite memory devices were prepared with the architecture of ITO/PEDOT:PSS/quasi-2D perovskite/Bphen $(20 \mathrm{~nm}) / \mathrm{Ag}$. The fabrication procedure of our symmetrical device can be found in our previous work. ${ }^{10}$ First, the $\mathrm{MAPbBr}_{3}$ film was spin-cast onto the ITO substrate to get the ITO/ $\mathrm{MAPbBr}_{3}$ unit. Second, two identical units were pasted face-to-face to form a configured device with the structure of ITO/MAPbBr $/$ $\mathrm{MAPbBr}_{3} / \mathrm{ITO}$. Finally, modulated voltage scans were applied to the device with a fixed pressure. All of the procedures were operated in a nitrogen-filled glovebox.

Characterizations and Measurements. The current-voltage characteristics were measured by a Keithley 2400. The film morphologies were characterized by SEM (Zeiss). The steady-state
PL characteristics were measured using a Flouro Log III spectrometer. Here, we use a femtosecond laser as the PL excitation, with a wavelength at $400 \mathrm{~nm}$, a repetition frequency of $1 \mathrm{kHz}$, and a spot size of around $100 \mu \mathrm{m}$ in diameter.

\section{ASSOCIATED CONTENT}

\section{Supporting Information}

The Supporting Information is available free of charge at https://pubs.acs.org/doi/10.1021/acsanm.0c02457.

List of several organic memory devices with the respective conductivity and switching types and additional $I-V$ curves, SEM images, EDS results, and schematic diagram (PDF)

\section{AUTHOR INFORMATION}

\section{Corresponding Author}

Jiajun Qin - State Key Laboratory of Surface Physics, Key Laboratory of Micro and Nano Photonic Structures (Ministry of Education) and Collaborative Innovation Center of Advanced Microstructures, Fudan University, Shanghai 200433, China; Department of Physics, Chemistry and Biology (IFM), Linköping University, Linköping SE-58183, Sweden; ㅇo이.org/0000-0002-9956-2358; Email: jiajun.qin@liu.se

\section{Authors}

Xianxi Yu - State Key Laboratory of Surface Physics, Key Laboratory of Micro and Nano Photonic Structures (Ministry of Education) and Collaborative Innovation Center of Advanced Microstructures, Fudan University, Shanghai 200433, China

Tangyao Shen - State Key Laboratory of Surface Physics, Key Laboratory of Micro and Nano Photonic Structures (Ministry of Education) and Collaborative Innovation Center of Advanced Microstructures, Fudan University, Shanghai 200433, China

Chunqin Zhu - State Key Laboratory of Surface Physics, Key Laboratory of Micro and Nano Photonic Structures (Ministry of Education) and Collaborative Innovation Center of Advanced Microstructures, Fudan University, Shanghai 200433, China

Qi Zeng - School of Material Engineering, Shanghai University of Engineering Science, Shanghai 201620, China

Anran Yu - State Key Laboratory of Surface Physics, Key Laboratory of Micro and Nano Photonic Structures (Ministry of Education) and Collaborative Innovation Center of Advanced Microstructures, Fudan University, Shanghai 200433, China

Shaobo Liu - State Key Laboratory of Surface Physics, Key Laboratory of Micro and Nano Photonic Structures (Ministry of Education) and Collaborative Innovation Center of Advanced Microstructures, Fudan University, Shanghai 200433, China

Ruichen Yi - State Key Laboratory of Surface Physics, Key Laboratory of Micro and Nano Photonic Structures (Ministry of Education) and Collaborative Innovation Center of Advanced Microstructures, Fudan University, Shanghai 200433, China; o orcid.org/0000-0001-6771-0718

Zhenhua Weng - Center for Micro Nano system, SIST, Fudan University, Shanghai 200433, China

Yiqiang Zhan - Center for Micro Nano system, SIST, Fudan University, Shanghai 200433, China 
Xiaoyuan Hou - State Key Laboratory of Surface Physics, Key Laboratory of Micro and Nano Photonic Structures (Ministry of Education) and Collaborative Innovation Center of Advanced Microstructures, Fudan University, Shanghai 200433, China; (1) orcid.org/0000-0002-4462-4566

Complete contact information is available at:

https://pubs.acs.org/10.1021/acsanm.0c02457

\section{Author Contributions \\ ${ }^{\perp}$ X.Y., T.S., and C.Z. contributed equally to this work. \\ Author Contributions}

J.Q. and X.H. directed the research project and supervised the experimental design. X.Y., T.S., and C.Z. conceived and performed the experimental studies and analyzed the data. Q.Z., A.Y., S.L., R.Y., Z.W., and Y.Z. contributed to the discussions. The manuscript was written by both J.Q. and X.H.

\section{Funding}

The National Natural Science Foundation of China (Grant 11574049).

\section{Notes}

The authors declare no competing financial interest.

\section{ACKNOWLEDGMENTS}

This research was supported by financial support from the National Natural Science Foundation of China (Grant 11574049) and the Natural Science Foundation of Shanghai (Grant 17ZR1402600).

\section{REFERENCES}

(1) Liu, M.; Johnston, M. B.; Snaith, H. J. Efficient Planar Heterojunction Perovskite Solar Cells by Vapour Deposition. Nature 2013, 501, 395-398.

(2) Zhang, J.; Wu, T.; Duan, J.; Ahmadi, M.; Jiang, F.; Zhou, Y.; Hu, B. Exploring Spin-orbital Coupling Effects on Photovoltaic Actions in $\mathrm{Sn}$ and Pb Based Perovskite Solar Cells. Nano Energy 2017, 38, 297303.

(3) Xu, W.; Hu, Q.; Bai, S.; Bao, C.; Miao, Y.; Yuan, Z.; Borzda, T.; Barker, A. J.; Tyukalova, E.; Hu, Z.; Kawecki, M.; Wang, H.; Yan, Z.; Liu, X.; Shi, X.; Uvdal, K.; Fahlman, M.; Zhang, W.; Duchamp, M.; Liu, J.-M.; Petrozza, A.; Wang, J.; Liu, L.-M.; Huang, W.; Gao, F. Rational Molecular Passivation for High-performance Perovskite Light-emitting Diodes. Nat. Photonics 2019, 13, 418-424.

(4) Wang, N.; Cheng, L.; Ge, R.; Zhang, S.; Miao, Y.; Zou, W.; Yi, C.; Sun, Y.; Cao, Y.; Yang, R.; Wei, Y.; Guo, Q.; Ke, Y.; Yu, M.; Jin, Y.; Liu, Y.; Ding, Q.; Di, D.; Yang, L.; Xing, G.; Tian, H.; Jin, C.; Gao, F.; Friend, R. H.; Wang, J.; Huang, W. Perovskite Light-emitting Diodes Based on Solution-processed Self-organized Multiple Quantum Wells. Nat. Photonics 2016, 10, 699-704.

(5) Qin, J.; Zhang, J.; Bai, Y.; Ma, S.; Wang, M.; Xu, H.; Loyd, M.; Zhan, Y.; Hou, X.; Hu, B. Enabling Self-passivation by Attaching Small Grains on Surfaces of Large Grains Toward High-performance Perovskite LEDs. iScience 2019, 19, 378-387.

(6) Dou, L.; Yang, Y.; You, J.; Hong, Z.; Chang, W.-H.; Li, G.; Yang, Y. Solution-processed Hybrid Perovskite Photodetectors with High Detectivity. Nat. Commun. 2014, 5, No. 5404.

(7) Fang, Y.; Dong, Q.; Shao, Y.; Yuan, Y.; Huang, J. Highly Narrowband Perovskite Single-crystal Photodetectors Enabled by Surface-charge Recombination. Nat. Photonics 2015, 9, 679-686.

(8) Jia, Y.; Kerner, R. A.; Grede, A. J.; Rand, B. P.; Giebink, N. C. Continuous-wave Lasing in an Organic-inorganic Lead Halide Perovskite Semiconductor. Nat. Photonics 2017, 11, 784-788.

(9) Xing, G.; Mathews, N.; Lim, S. S.; Yantara, N.; Liu, X.; Sabba, D.; Grätzel, M.; Mhaisalkar, S.; Sum, T. C. Low-temperature Solutionprocessed Wavelength-tunable Perovskites for Lasing. Nat. Mater. 2014, 13, 476-480.
(10) Qin, J.; Zhang, J.; Chen, X.; Zeng, Q.; Peng, H.; Feng, W.; Tang, Y.; Hou, X. TOP-electrode-eliminated Organic Bi-stable Devices and Their two Switching Modes in Different Atmospheres. Org. Electron. 2015, 22, 127-131.

(11) Qin, J.; Chu, M.; Peng, H.; Zhang, J.; Hou, X. Square Wave Voltages-induced ON States of Organic Resistive Memory Devices. Appl. Phys. Lett. 2016, 109, No. 153303.

(12) Chang, S. H.; Lee, J. S.; Chae, S. C.; Lee, S. B.; Liu, C.; Kahng, B.; Kim, D. W.; Noh, T. W. Occurrence of Both Unipolar Memory and Threshold Resistance Switching in a NiO Film. Phys. Rev. Lett. 2009, 102, 26801.

(13) Kim, H.; An, H.; Lee, E. B.; Kim, T. G. Stable Bipolar Resistive Switching Characteristics and Resistive Switching Mechanisms Observed in Aluminum Nitride-based ReRAM Devices. IEEE Trans. Electron Devices 2011, 58, 3566-3573.

(14) Möller, S.; Perlov, C.; Jackson, W.; Taussig, C.; Forrest, S. R. A Polymer/semiconductor Write-once Read-many-times Memory. Nature 2003, 426, 166-169.

(15) Teo, E. Y. H.; Ling, Q. D.; Song, Y.; Tan, Y. P.; Wang, W.; Kang, E. T.; Chan, D. S. H.; Zhu, C. Non-volatile WORM Memory Device Based on an Acrylate Polymer with Electron Donating Carbazole Pendant Groups. Org. Electron. 2006, 7, 173-180.

(16) Gu, C.; Lee, J.-S. Flexible Hybrid Organic-inorganic Perovskite Memory. ACS Nano 2016, 10, 5413.

(17) Bakaul, S. R.; Serrao, C. R.; Lee, O.; Lu, Z.; Yadav, A.; Carraro, C.; Maboudian, R.; Ramesh, R.; Salahuddin, S. High Speed Epitaxial Perovskite Memory on Flexible Substrates. Adv. Mater. 2017, 29, No. 1605699.

(18) Tian, H.; Zhao, L.; Wang, X.; Yeh, Y.-W.; Yao, N.; Rand, B. P.; Ren, T.-L. Extremely Low Operating Current Resistive Memory Based on Exfoliated 2D Perovskite Single Crystals for Neuromorphic Computing. ACS Nano 2017, 11, 12247.

(19) Wu, Y.; Wei, Y.; Huang, Y.; Cao, F.; Yu, D.; Li, X.; Zeng, H. Capping $\mathrm{CsPbBr}_{3}$ with $\mathrm{ZnO}$ to Improve Performance and Stability of Perovskite Memristors. Nano Res. 2017, 10, 1584-1594.

(20) Cao, X.; Han, Y.; Zhou, J.; Zuo, W.; Gao, X.; Han, L.; Pang, X.; Zhang, L.; Liu, Y.; Cao, S. Enhanced Switching Ratio and Long-term Stability of Flexible RRAM by Anchoring Polyvinylammonium on Perovskite Grains. ACS Appl. Mater. Interfaces 2019, 11, 3591435923.

(21) Wang, Y.; Lv, Z.; Liao, Q.; Shan, H.; Chen, J.; Zhou, Y.; Zhou, L.; Chen, X.; Roy, V. A.; Wang, Z.; et al. Synergies of Electrochemical Metallization and Valance Change in All-inorganic Perovskite Quantum Dots for Resistive Switching. Adv. Mater. 2018, 30, No. 1800327.

(22) Roy, A.; Jang, H. W.; Cha, P.-R. Effects of Mobile Charged Defects on Current-voltage Behavior in Resistive Switching Memories Based on Organic-inorganic Hybrid Perovskite. Appl. Phys. Lett. 2018, 113, No. 193301.

(23) Han, J. S.; Le, Q. V.; Choi, J.; Hong, K.; Moon, C. W.; Kim, T. L.; Kim, H.; Kim, S. Y.; Jang, H. W. Air-stable Cesium Lead Iodide Perovskite for Ultra-low Operating Voltage Resistive Switching. Adv. Funct. Mater. 2018, 28, No. 1705783.

(24) Kang, K.; Ahn, H.; Song, Y.; Lee, W.; Kim, J.; Kim, Y.; Yoo, D.; Lee, T. High-performance Solution-processed Organo-metal Halide Perovskite Unipolar Resistive Memory Devices in a Cross-bar Array Structure. Adv. Mater. 2019, 31, No. 1804841.

(25) Yoo, E. J.; Lyu, M.; Yun, J.-H.; Kang, C. J.; Choi, Y. J.; Wang, L. Resistive Switching Behavior in Organic-inorganic Hybrid $\mathrm{CH}_{3} \mathrm{NH}_{3} \mathrm{PbI}_{3-\mathrm{x}} \mathrm{Cl}_{\mathrm{x}}$ Perovskite for Resistive Random Access Memory Devices. Adv. Mater. 2015, 27, 6170-6175.

(26) Lee, S.; Kim, H.; Kim, D. H.; Kim, W. B.; Lee, J. M.; Choi, J.; Shin, H.; Han, G. S.; Jang, H. W.; Jung, H. S. Tailored 2D/3D Halide Perovskite Heterointerface for Substantially Enhanced Endurance in Conducting Bridge Resistive Switching Memory. ACS Appl. Mater. Interfaces 2020, 12, 17039-17045.

(27) You, Y. T.; Wang, M. L.; Xuxie, H. N.; Wu, B.; Sun, Z. Y.; Hou, X. Y. Conductance-dependent Negative Differential Resistance in Organic Memory Devices. Appl. Phys. Lett. 2010, 97, 261. 
(28) Zhang, J.; Qin, J.; Wang, M.; Bai, Y.; Zou, H.; Keum, J. K.; Tao, R.; Xu, H.; Yu, H.; Haacke, S.; Hu, B. Uniform Permutation of Quasi2D Perovskites by vacuum poling for Efficient, High-fill-factor Solar Cells. Joule 2019, 3, 3061-3071.

(29) Qiu, L.; He, S.; Ono, L. K.; Qi, Y. Progress of Surface Science Studies on $\mathrm{ABX}_{3}$-based Metal Halide Perovskite Solar Cells. Adv. Energy Mater. 2020, 10, No. 1902726.

(30) Belisle, R. A.; Bush, K. A.; Bertoluzzi, L.; Gold-Parker, A.; Toney, M. F.; McGehee, M. D. Impact of Surfaces on Photoinduced Halide Segregation in Mixed-halide Perovskites. ACS Energy Lett. 2018, 3, 2694-2700.

(31) Cho, B.; Yun, J. M.; Song, S.; Ji, Y.; Kim, D. Y.; Lee, T. Direct Observation of Ag Filamentary Paths in Organic Resistive Memory Devices. Adv. Funct. Mater. 2011, 21, 3976-3981. 